\title{
The interventions to improve standard precautions (sp) knowledge and practice among healthcare workers (hcws): a systematic review.
}

\begin{abstract}
Background: Standard Precautions (SP) is the basic requirement in infection control measures to reduce healthcare-associated infections (HAIs) as well occupational infection in healthcare settings. Many interventions have been done to increase the knowledge and practice of SP among healthcare workers (HCWs).

Objectives: To assess the effectiveness of interventions to improve knowledge and practice on Standard Precautions among HCWs.

Materials and Methods: Articles were searched through Pubmed, Medline, Scopus and Google Scholar search engines using keywords "Standard Precautions OR infection control AND interventional study OR experimental study". Types of studies included are randomised control trials of individuals, cluster-randomised trials, non-randomised trials, controlled before-after studies, and cross sectional study. Only articles written in English published from 2010 to 2018 and accessible as free full texts were included in this review. The articles must evaluate the multiple components of Standard Precautions simultaneously.

Result: There are 14 articles included in this study. Most of the studies implemented educational and training intervention with or without infection control support. Other intervention strategies were theory-based module, self-instructed computer module with clinical case simulation, checklist and coloured cues for communication enhancement as well as Healthcare-Associated Infection Prevention Initiative (CHAIPI). However, different studies used different measures to assess the knowledge and practice of Standard Precautions among HCWs.

Conclusion: Because of this heterogeneity, it is difficult to draw a clear conclusion about the effectiveness of different interventions. However, it was shown that interventions do promote Standard Precautions knowledge and practice, but further research is warranted to determine which interventions are the most effective.
\end{abstract}

Keyword : Standard precautions; Intervention; Healthcare workers; Knowledge; Practice. 